\title{
Skeletal muscle low attenuation area and maximal fat oxidation rate during submaximal exercise in male obese individuals
}

\author{
O Dériaz et al \\ International Journal of Obesity (2002) 26, 1406. doi:10.1038/sj.ijo.0802031 \\ Correction to: International Journal of Obesity (2001) 25, 1579-1584
}

Following the publication of the above paper, the author had identified errors in the abstract and also within Table 2. The correct version of the Abstract and Table 2 is reproduced below.

BACKGROUND: Muscle triacylglycerols (TG) are known to be a source of energy during submaximal exercise.

OBJECTIVE: The aim of this study was to assess whether an index of muscle fat content is related to maximal fat oxidation rate $\left(\right.$ FATOX $_{\max }$ ) in 58 obese men (mean age: $46.0 \pm 0.8$ (s.e.) y, body weight: $96.4 \pm 1.4 \mathrm{~kg}$, percentage fat: $31.9 \pm 0.5 \%$ ).

DESIGN: FATOX $_{\max }$ was defined as the highest value of fat oxidation rate, measured by indirect calorimetry, while walking on a treadmill $(4.3 \mathrm{~km} / \mathrm{h})$ at three different slopes: $0 \%\left(40 \pm 1 \%\right.$ of $\left.\mathrm{VO}_{2 \max }\right), 3 \%\left(47 \pm 1 \%\right.$ of $\left.\mathrm{VO}_{2 \max }\right)$ and $6 \%\left(58 \pm 1 \%\right.$ of $\left.\mathrm{VO}_{2 \max }\right)$. Fat-free mass (FFM) and fat mass (FM) were measured with the underwater technique and scans were obtained by computed tomography (CT) at the mid thigh level to assess areas of adipose tissue within skeletal muscle, ie deep adipose tissue (DAT), subcutaneous adipose tissue (SAT), skeletal muscle (M) and low attenuation skeletal muscle (LAM, range of attenuation values: $0-34$ Hounsfield units). LAM and DAT were used as indices of skeletal muscle fat content.

RESULTS: FATOX ${ }_{\text {max }}$, adjusted for age, was correlated with FFM $(r=0.26, P=0.05)$, LAM $(r=0.29, P<0.05)$, abdominal visceral adipose tissue $(r=0.30, P<0.05)$ and plasma free fatty acid levels $(r=0.33, P<0.05)$ but not with SAT $(r=0.03)$ and DAT $(r=0.21)$. In a stepwise linear multiple regression, plasma FFA, age and LAM significantly predicted FATOX $\max _{\max }\left(r^{2}=0.27\right)$. Each independent variable explained about $9 \%$ of the FATOX ${ }_{\max }$ variance.

CONCLUSION: LAM makes a significant but weak contribution to the modulation of fat oxidation during submaximal exercise in obese men.

Table 2 Pearson coefficients between FATOX ${ }_{\max }, \mathrm{VO}_{2 \max }$ and several potential predictors

\begin{tabular}{|c|c|c|c|}
\hline Variable & Adjusted FATOX $\max ^{a}$ & Adjusted FATOX $_{\max }^{\mathrm{b}}$ & Adjusted $\mathrm{VO}_{2 \max }{ }^{\mathrm{a}}$ \\
\hline Adjusted FATOX ${ }_{\max }{ }^{a}$ & 1.000 & 0.964 & $0.368^{*}$ \\
\hline Adjusted FATOX ${ }_{\max }{ }^{b}$ & $0.963^{*}$ & 1.000 & $0.314^{*}$ \\
\hline Adjusted $\mathrm{VO}_{2 \max }{ }^{\mathrm{a}}$ & $0.388^{*}$ & $0.314^{*}$ & 1.000 \\
\hline LAM & $0.294^{*}$ & 0.178 & $0.314^{*}$ \\
\hline Abdominal AT visceral & $0.299^{*}$ & 0.188 & -0.012 \\
\hline Abdominal AT subcutaneous & 0.177 & -0.035 & 0.168 \\
\hline FFM & 0.257 & 0.125 & $0.483^{*}$ \\
\hline FM & $0.266^{*}$ & 0.000 & 0.237 \\
\hline Fasting FFA & $0.325^{\star}$ & $0.330^{*}$ & -0.071 \\
\hline Thigh skeletal muscle & 0.132 & 0.098 & 0.189 \\
\hline Thigh AT subcutaneous & 0.027 & -0.175 & 0.149 \\
\hline Thigh AT deep & 0.211 & 0.098 & 0.213 \\
\hline
\end{tabular}

